\title{
FAMILY-BASED ASSOCIATION STUDY OF KILLER CELL IMMUNOGLOBULIN-LIKE RECEPTOR GENES WITH LEUKEMIA
}

\author{
V. Varbanova', S. Mihaylova ${ }^{2}$, E. Naumova², C. Cotoc ${ }^{3}$, A. Mihaylova² \\ ${ }^{1}$ Clinic of Hematology - Military Medical Academy, MHAT, Sofia, Bulgaria \\ 2Department of Clinical Immunology and Stem Cell Bank, University Hospital "Alexandrovska", \\ Medical University - Sofia, Bulgaria \\ ${ }^{3}$ College of Applied Health Sciences, University of Illinois, USA
}

\begin{abstract}
NK cell function is controlled by the cell expression of killer immunoglobulin-like receptors (KIRs) and their ligation with the corresponding HLA ligands. Various malignancies have been associated with certain KIRs surface cell expression and various KIR/ HLA ligand combinations. Prior research using case/control study design demonstrates the role of KIR and KIR HLA ligands as genetic factor involved in tumor susceptibility. The objective of this study was to investigate the family-based association of KIRs, HLA class I ligands and KIR/ligand combinations with leukemia diagnosis in families having a leukemia diagnosed child. Sixty-seven families that met the index leukemia case criteria (acute lymphoblastic leukemia, $A L L, n=45$; acute myeloid leukemia, $A M L, n=13$; chronic myeloid leukemia, CML, $n=9$; first degree healthy relatives $n=159$ ) were examined. Our study consisted of two phases. In Phase1 case-control study, we primarily compared patients to their healthy siblings to asses if a marker or genotype may be associated with leukemia, excluding the impact of the environment. Phase 2 consisted of a secondary family-based association study. KIR genotyping was performed by PCR-SSP method. KIR $H L A$ ligands were defined by direct method using PCR-SSP method and/or indirect base on high resolution typing of HLA-A, $-B,-C$ alleles. Results of phase 1 showed an increase in the frequency of KIR genotype (with a ratio $=0.57$; higher frequency for inhibitory KIRs vs. activating KIRs) among leukemia patients compared to healthy siblings. Results of the phase 2 familial study observed an association between HLA-C1+/BBw4 $+/ A^{B w 4}+$ haplotype (a mediator of inhibitory signals) and leukemia. Also, we concluded that the absence of $H L A-A^{B w 4}$ alleles was related to leukemia development.
\end{abstract}

Key words: KIR, HLA class I ligand, leukemia, family-based study

Corresponding author: Viktoria Plamenova Varbanova, Clinic of Hematology, Military Medical Academy, Multiprofile Hospital for Active Treatment, 3 Sv. Georgi Sofiyski Blvd., 1606 Sofia, Bulgaria, Ph: +359884 674160, e-mail: viktoriia1982@abv.bg

\section{INTRODUCTION}

T here is potential for transformation of normal cells in tumor cells in each organism. In healthy individuals, tumor transformed cells are destroyed by the self-immune system. NK cells play an important role as first line in self- immune defense. Growing evidence suggests the relevance of NK cell surface receptors members of the immunoglobulin superfamily, particularly KIR and corresponding KIR ligands in tumor development and susceptibility [1]. So far $16 \mathrm{KIR}$ genes and $907 \mathrm{KIR}$ alleles and en- 
coding KIRs have been described [1]. HLA class I molecules act as specific ligands for some of these receptors [2-10].

HLA-C1 ligand group (serine and asparagine residues at positions 77 and 80 in the alpha 1 helix of the MHC class I heavy chain) binds specific KIR2DL1, while $\mathrm{C} 2$ allotypes (asparagine and lysine residues at the same positions) bind KIR2DL2/2DL3 [2, 3]. HLA$A$ and $B$ alleles with Bw4 epitope act as ligands for KIR3DL1 [3, 4]. The strength of KIR/ligand binding depends on the amino acid at position 80 in Bw4 molecules. Bw4 alleles with isoleucine $\left(B w 4^{180}\right)$ are more potent ligands for KIRs in comparison to Bw4 alleles with threonine $\left(B w 4^{T 80}\right)$ at this position [5]. KIR3DL2 recognizes HLA-A3 and HLA-A11 with selected peptides like EBV-encoded peptide [6]. The activating KIRs: 2DS1, 2DS2 and 3DS1 are thought to share HLA ligand binding specificities with their inhibitory counterparts: KIR2DL1, 2DL2/2DL3 and 3DL1, respectively. KIR2DS1 binds weakly to HLA-C2 allotypes [7], KIR2DS2 may interact weakly to HLA-C1 [7] and KIR3DS1 with HLA-Bw4 molecules [9, 10].

Recently, considerable data from case/controls studies demonstrate the role of KIR and KIR HLA ligands as genetic factor involved in tumor susceptibility [1]. It could be implied that genetically predetermined $\mathrm{KIR} / \mathrm{HLA}$ combinations in terms of stronger activation or lower inhibitory potential influence NK antitumor activity and malignancy susceptibility [11]. As such, KIR-mediated activation due to activating receptors KIR3DS1, KIR2DS3 and KIR2DS1 was associated with protection from hematology malignancies [12-14]. Similarly, genotypes with inhibitory potential are presented as risk factors for tumor development as KIR2DL2/2DL3/HLA-C1 in leukemia patients [15]. However, additional data contradicts the hypothesis of KIR2DS1 [16-18], KIR2DS3 [17, 18], KIR2DS2 and KIR2DS5's as predisposing mediating activation effect for leukemia [18]. Nevertheless, none of the prior studies considered the environmental factors, which also influence tumor development. We found only two prior studies evaluating $\mathrm{KIR} / \mathrm{HLA}$ polymorphisms in the context of oncohematological diseases [13, 19]. Besson et al's work [13] demonstrates the activating receptors KIR3DS1 and KIR2DS1 as protective factors in a familial study of Hodgkin's lymphoma. Sugioka et al. [19] report KIR2DL2, KIR2DL5, KIR2DS1, KIR2DS2 and KIR2DS3 as being protective in a group of patients with various hematological diseases compared to healthy family members.

Thus, we hypothesized that a family-based study will determine whether there are genetic differences that act as predetermined predisposing factors for leuke- mia development, assuming that leukemia affected and non-affected siblings were exposed to similar environmental factors.

\section{MATERIAL AND METHODS}

\section{Study population}

Sixty-seven families were included that met the index leukemia case. Patients study sample consisted of ALL ( $n=45,30$ males and 15 females; average age $19 \pm 10.1$ years), AML ( $n=13,7$ males and 6 females; average age $35 \pm 13.5$ years) and CML ( $\mathrm{n}$ $=9,7$ males and 2 females; average age $38.4 \pm 12.1$ years). First degree healthy relatives (97 parents and 62 siblings) were included in the study after an interview in order to elicit their medical history. Written and informed consent was obtained from parents and written and informed assent was obtained from children participants. The study was approved by the local ethics committee and IRB boards.

\section{Methods}

\section{Measures}

DNA was extracted from peripheral blood using iPrep PureLink $^{\circledast}$ gDNA $^{\text {TM }}$ Blood kit (Invitrogen, USA) and iPrep $^{\mathrm{TM}}$ Purification instrument (Invitrogen, USA).

KIR genotyping was performed by PCR-SSP method with commercially available Olerup SSP ${ }^{\circledR}$ KIR typing kit (Olerup SSP AB, Sweden). Briefly 24 locus specific primer sets in KIR genotyping kit allow detection of $16 \mathrm{KIR}$ genes and pseudogenes and discrimination of KIR2DL5A, KIR2DL5B, KIR3DL1*004 alleles and two groups KIR2DS4 alleles (group $1-K I R 2 D S 4^{*} 001$ from group $\left.2-K I R 2 D S 4^{*} 003 / 004 / 006 / 007\right)$. The results obtained were interpreted using the worksheet provided by the manufacturer.

KIR HLA Ligands were determined using direct genotyping by commercially available Olerup SSP ${ }^{\circledR} \mathrm{KIR}$ HLA ligand typing kit (Olerup SSP AB, Sweden) and/ or indirect using KIR ligand calculator [20] base on high resolution typing of HLA-A, -B, -C alleles (AlleleSEQR HLA-A, -B and -C PCR/Sequencer Kit, Atria Genetics, USA) as previously described [21]. KIR HLA ligand typing kit use 5 primers sets to distinguish HLA-C1, $-\mathrm{C} 2$, $-\mathrm{Bw} 4^{180},-\mathrm{Bw} 4^{\mathrm{T} 80}$ and $-\mathrm{A}^{\mathrm{Bw} 4}+$ ligand group. The results obtained were interpreted using the worksheet provided by the manufacturer. HLA-A3 and HLA-A11 was determined based on individual HLA-A genotype, which was examined by OIerup SSP ${ }^{\circledR}$ "HLA-A low resolution" (Olerup SSP AB, Sweden) or by AlleleSEQR HLA-A PCR/Sequencer Kit (Atria Genetics, USA) according to the manufacture's instruction. 
KIR gene, HLA class I ligand group and KIR/HLA Iigand combination frequencies were estimated by direct counting. Individual KIR haplotype and KIR genotype were defined according to the allele frequency net database (AFND) [1, 22].

\section{Data Analysis}

In the present study we used two models for comparative analysis.

Phase 1: In the case/control study we compared carrier frequencies, in terms of the presence or absence of KIR genes, their HLA ligands and KIR/HLA ligand combinations between patients and their healthy siblings using Pearson's chi-squared test and Fisher exact test. Also, KIR haplotype and KIR genotype frequencies were included in the analysis. Odds ratios (ORs) with $95 \%$ confidence intervals (Cls) were calculated for variables with statistical significance. Statistical analysis was performed using SPSS for Windows, version 16.0 (SPSS Inc., Chicago, IL).

Phase 2: In the family-base study, a $2^{\text {nd }}$ analysis with "transmission disequilibrium test" (TDT) was conducted [23]. Measures of pairwise linkage disequilibrium (LD) between KIR genes in, such as D' and r2 [24], were estimated using Haploview (http://www. broad.mit.edu/personl/jcbarret/haplo/) according to the model of Besson et al [13]. |D'| and $r 2$ varied between 0 and 1. The TDT analysis was used to evaluate if there is distortion in the trasmmission of alleles of interest (KIR and KIR HLA class I ligands) from parents to affected offspring. TDT was performed after genotypic reconstruction for each KIR gene, using all first-degree members of the families. In cases were one parent was missing (18/67 in our sample) families can be analysed either by method of Knapp
M (RC-TDT) [25] or by method of Spielman RS (SibTDT) [26]. The family-based association study was conducted by FBAT program [27], in which these three described methods for genotype reconstruction are implicated (TDT, RC-TDT, and Sib-TDT).

\section{RESULTS}

\section{Phase 1. Case-control association study}

\section{KIR gene, haplotype and genotype frequencies}

The presence of the $16 \mathrm{KIR}$ genes (14 genes and 2 pseudogenes) in patients and their healthy siblings are presented in Table 1.

The frequency of the KIR genotypes (presence/ absence of KIR gene) in each of the two groups is showed in Table 2.

In both patients and non-affected brothers/sisters KIR genotype ID1 was the most frequent, which corresponds to homozygous carrier of two KIRA haplotypes (KIRAA). No other KIRAA haplotype combination was found, so the frequency of KIRAA in each group was relevant to KIR genotype ID1 rate $(21.4 \%$ and $29.0 \%$ respectively). All other individuals were KIRBx (KIRBB or KIRAB) (Table 2).

Although no significant difference in frequency of KIR genes, genotypes and haplotype combinations were found when patients and healthy siblings were compared, we analysed the frequency of genotypes with respect to the ratio of the number of activating to inhibitory KIR genes calculated for all individuals according to the model of Karabon et al. [12]. In this approach KIR2DL4 was included in activating KIR group (the transmembrane arginine near extracellular domain has predominantly activating function $[28,29])$.

Table 1. Frequencies of KIR genes in leukemia patients $(n=56)$ and their healthy siblings $(n=62)$

\begin{tabular}{|l|c|c|c|}
\hline & Patients $\mathbf{n}(\%)$ & Healthy siblings $\mathbf{n}(\%)$ & $\mathbf{p}$ \\
\hline KIR2DL1 & $54(96.4)$ & $61(98.4)$ & $\mathrm{ns}$ \\
\hline KIR2DL2 & $40(71.4)$ & $39(62.9)$ & $\mathrm{ns}$ \\
\hline KIR2DL3 & $46(82.1)$ & $53(85.5)$ & $\mathrm{ns}$ \\
\hline KIR2DL5 & $36(64.3)$ & $36(58.1)$ & $\mathrm{ns}$ \\
\hline KIR3DL1 & $52(92.9)$ & $59(95.2)$ & $\mathrm{ns}$ \\
\hline KIR2DS1 & $24(42.9)$ & $23(37.1)$ & $\mathrm{ns}$ \\
\hline KIR2DS2 & $40(71.4)$ & $33(62.9)$ & $\mathrm{ns}$ \\
\hline KIR2DS3 & $27(48.2)$ & $29(46.8)$ & $\mathrm{ns}$ \\
\hline KIR2DS4 & $53(94.6)$ & $59(95.1)$ & $\mathrm{ns}$ \\
\hline KIR2DS5 & $24(42.9)$ & $19(30.7)$ & $\mathrm{ns}$ \\
\hline KIR3DS1 & $24(42.9)$ & $24(38.7)$ & $\mathrm{ns}$ \\
\hline KIR3DL2/KIR3DL3/KIR2DL4 & $56(100)$ & $62(100)$ & $\mathrm{ns}$ \\
\hline
\end{tabular}


Table 2. Frequency of KIR genotypes in leukemia patients $(n=56)$ and their healthy siblings $(n=62)$

\begin{tabular}{|c|c|c|c|c|c|c|c|c|c|c|c|c|c|c|c|c|c|}
\hline $\begin{array}{l}\text { Patients } \\
\mathrm{n}(\%)\end{array}$ & $\begin{array}{c}\text { Healthy sibs } \\
\mathrm{n}(\%)\end{array}$ & $I D^{*}$ & 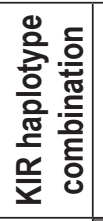 & Б्户 & בั & ล) & 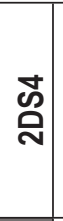 & วิ & 吕 & क्ल & হू & กิ & ח̊ & مٌ & 芩 & ì & 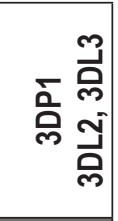 \\
\hline $12(21.4)$ & $18(29.0)$ & 1 & AA & & & & & & & & & & & & & & \\
\hline $7(12.5)$ & $8(12.9)$ & 5 & $\mathrm{Bx}$ & & & & & & & & & & & & & & \\
\hline $6(10.7)$ & $5(8.2)$ & 4 & $\mathrm{Bx}$ & & & & & & & & & & & & & & \\
\hline $2(3.6)$ & $3(4.8)$ & 2 & $\mathrm{Bx}$ & & & & & & & & & & & & & & \\
\hline $8(14.2)$ & $8(12.9)$ & 6 & $\mathrm{Bx}$ & & & & & & & & & & & & & & \\
\hline $4(7.1)$ & $4(6.5)$ & 3 & $\mathrm{Bx}$ & & & & & & & & & & & & & & \\
\hline $3(5.4)$ & $4(6.5)$ & 71 & $\mathrm{Bx}$ & & & & & & & & & & & & & & \\
\hline $4(7.1)$ & $2(3.2)$ & 73 & $\mathrm{Bx}$ & & & & & & & & & & & & & & \\
\hline $1(1.8)$ & $1(1.6)$ & 69 & $\mathrm{Bx}$ & & & & & & & & & & & & & & \\
\hline $2(3.6)$ & $1(1.6)$ & 72 & $\mathrm{Bx}$ & & & & & & & & & & & & & & \\
\hline $1(1.8)$ & $1(1.6)$ & 81 & $\mathrm{Bx}$ & & & & & & & & & & & & & & \\
\hline $2(3.6)$ & 0 & 9 & $\mathrm{Bx}$ & & & & & & & & & & & & & & \\
\hline $2(3.6)$ & 0 & 13 & $\mathrm{Bx}$ & & & & & & & & & & & & & & \\
\hline $1(1.8)$ & 0 & 150 & $\mathrm{Bx}$ & & & & & & & & & & & & & & \\
\hline $1(1.8)$ & 0 & 70 & $\mathrm{Bx}$ & & & & & & & & & & & & & & \\
\hline 0 & $3(4.8)$ & 7 & $\mathrm{Bx}$ & & & & & & & & & & & & & & \\
\hline 0 & $1(1.6)$ & 22 & $\mathrm{Bx}$ & & & & & & & & & & & & & & \\
\hline 0 & $1(1.6)$ & 62 & $\mathrm{Bx}$ & & & & & & & & & & & & & & \\
\hline 0 & $1(1.6)$ & 90 & $\mathrm{Bx}$ & & & & & & & & & & & & & & \\
\hline 0 & $1(1.6)$ & 104 & $\mathrm{Bx}$ & & & & & & & & & & & & & & \\
\hline
\end{tabular}

The filled squares correspond to the presence of the KIR gene and the empty squares - to lack of the corresponding KIR gene; * Identification number defined according to http://www.allelefrequencies.net/kir6001a.asp

Only Group 1 KIR2DS4 alleles (2DS4*001) positive individuals accounted as positive for this activating KIR gene. Group 2 KIR2DS4 alleles (included the deleted 2DS4*003, $\left.{ }^{*} 004\right)$ were not functional. Ratios ranged from 0.2 (prevalence of inhibitory KIR genes) to 1.20 (overrepresentation of activating KIR genes).
KIR genotype corresponding to ratio 0.57 (seven inhibitory-KIR genes and four activating-KIR genes) was found to have a tendency for higher frequency among patients than among their healthy brothers/ sisters $(14.3 \%$ vs $4.8 \%, p=0.078, \mathrm{OR}=2.41[95 \% \mathrm{Cl}$ 0.73-16.6]) (Table 3).

Table 3. KIR genotypes ration in leukemia patients $(n=56)$ and their healthy siblings $(n=62)$

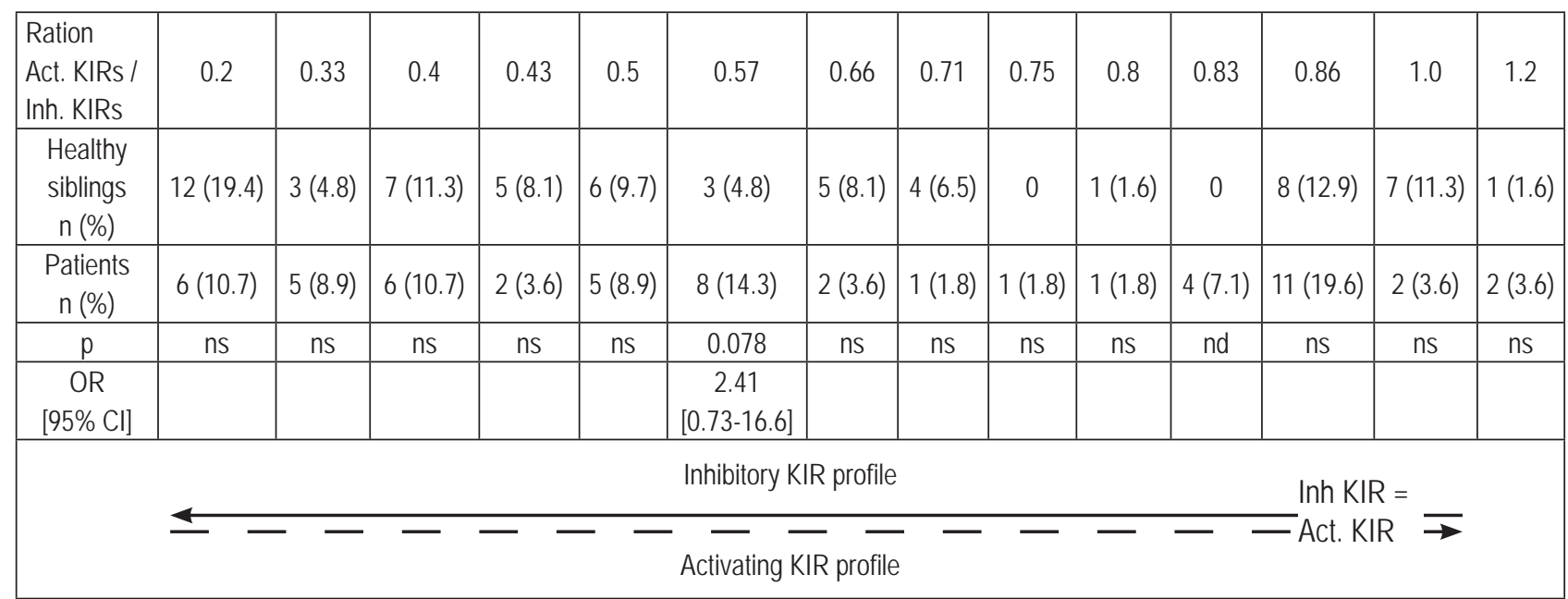

Abbr.: ns - not significant, nd - not defined, act. KIR - activating KIR; inh. KIR - inhibitory KIR; [95\% Cl] confidencial interval 


\section{Frequencies of KIR ligands and KIR/ligand combinations}

Not significant associations were observed comparing leukemia patients and their healthy siblings according to the frequencies of KIR HLA class I ligands and HLA class I ligand genotypes (Table 4).

Table 4. Frequencies of KIR ligands and KIR/Ligands combinations in leukemia patients $(n=56)$ and their healthy siblings $(n=62)$

\begin{tabular}{|c|c|c|c|}
\hline & $\begin{array}{c}\text { Patients } \\
\mathrm{n}(\%)\end{array}$ & $\begin{array}{c}\text { Healthy siblings } \\
\mathrm{n}(\%)\end{array}$ & $p$ \\
\hline HLA-C1 & $43(76.8)$ & $45(72.5)$ & ns \\
\hline HLA-C2 & $38(65.9)$ & $40(64.5)$ & ns \\
\hline HLA-C1C1 & $18(32.1)$ & $22(35.5)$ & ns \\
\hline $\mathrm{HLA}-\mathrm{C} 2 \mathrm{C} 2$ & $13(23.2)$ & $17(27.4)$ & $\mathrm{ns}$ \\
\hline HLA-C1C2 & $25(44.6)$ & $23(37.1)$ & ns \\
\hline HLA-Bw6 & $43(76.8)$ & $50(80.6)$ & ns \\
\hline HLA-Bw4 & $38(67.9)$ & $51(66.1)$ & ns \\
\hline HLA- Bw4 ${ }^{180}$ & $30(53.6)$ & $34(54.8)$ & ns \\
\hline HLA-Bw $4^{\text {T80 }}$ & $11(19.6)$ & $10(16.1)$ & ns \\
\hline HLA- Bw4 $4^{180} B w 4^{T 80}$ & $2(3.6)$ & $3(4.8)$ & ns \\
\hline HLA-Bw4 ${ }^{180} B w 4^{180}$ & $7(12.5)$ & $7(11.3)$ & ns \\
\hline $\mathrm{HLA}-\mathrm{B} w 4^{\mathrm{T} 80} \mathrm{Bw} \mathbf{4}^{\mathrm{T} 80}$ & $4(7.1)$ & $2(3.2)$ & $\mathrm{ns}$ \\
\hline $\mathrm{HLA}-\mathrm{A}^{\mathrm{BW4}+}$ & $25(44.6)$ & $26(42.0)$ & $\mathrm{ns}$ \\
\hline HLA- A3/11 & $13(23.2)$ & $18(29.1)$ & $\mathrm{ns}$ \\
\hline
\end{tabular}

The frequencies of inhibitory KIR/ligand combinations and the activating counterpart as well as KIR inhibitory/KIR activating countrpart/appropriate ligand between the two groups are presented in Table 4.
There were no statistically significant differences in the frequencies of KIR/ligand genotypes between the compared groups.

\section{Phase 2: Family-based study}

\section{Genotype reconstruction in families}

Genotypic reconstruction in families was carried out according to the Mendelian laws of inheritance and the model described by Besson et al. [13]. The procedure consisted of two steps. In the first step the following rules were followed:

1. Individuals missing particular KIR were determined as negative for the two alleles of this gene, for example KIR2DS5-/KIR2DS5-.

\section{KIR2DL2/2DL3 and KIR3DL1/3DS1 segregate as} alleles.

3. The used KIR genotyping kit could discriminate two allele groups of KIR2DL5 (2DL5A and 2DL5B) and KIR2DS4 (group 1 and group 2), which helped in the segregation analysis.

4. The framework KIR genes were excluded.

5. Like the framework KIR genes, KIR2DL1 was ubiquitous distributed (96\%) and also was excluded.

This step allowed us to determine $100 \%$ of KIR2DL2/2DL3 and KIR3DL1/3DS1 alleles and more than $50 \%$ of the remaining KIR genotypes (number of $\mathrm{KIR}$ alleles) in the analysis families. In the next step we used the known KIR genotypes for estimation of pairwise LD between KIRs based on the genotypes determined in this first reconstruction step (Figure 1).

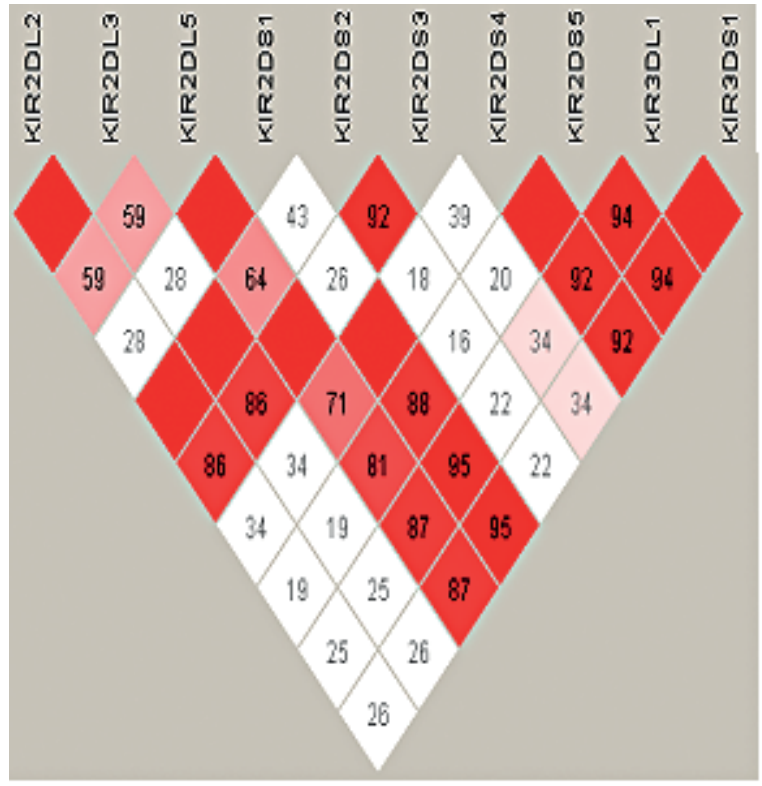

B

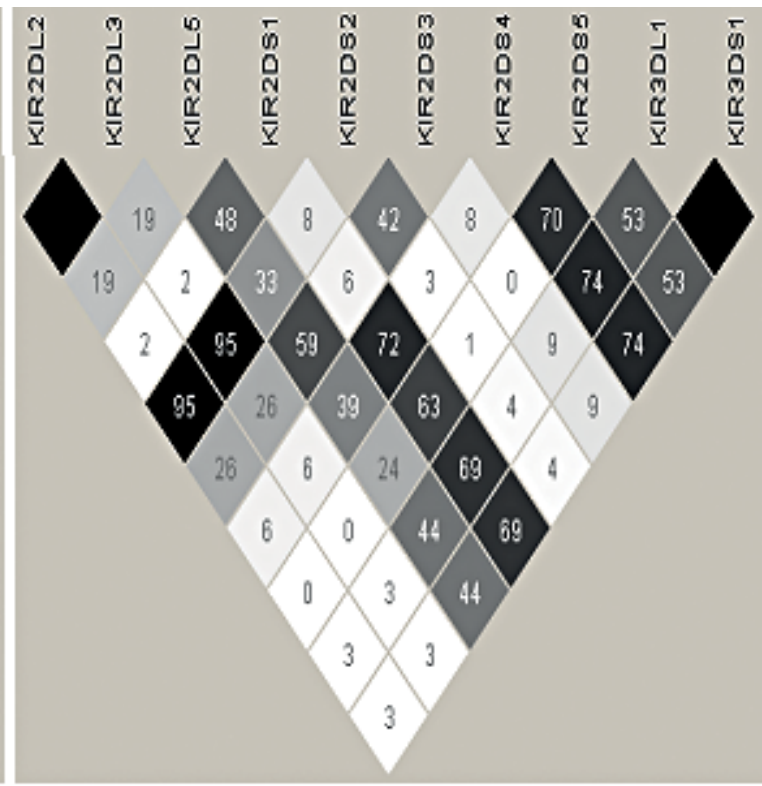

Fig. 1. Pairwise linkage disequilibrium between KIR genes in the family sample. Each square represents the magnitude of pairwise LD between KIR genes, as measured by D' (panel A) or r2 (panel B). Red squares represent a |D'| and black squares represent r2 value of 1.0 , corresponding to complete and perfect LD, respectively. All other colours in both figure indicate |D'| or R2 values < 0.8 
KIR region was in strong $L D$, and three pairs of KIRs were found to be in perfect $L D(r 2=1)$. KIR2DL2 and KIR2DS2 were in perfect positive LD thus KIR2DL2+/ KIRDS2+ and KIR2DL2-/KIRDS2- were the only haplotypes observed. By analogy KIR2DL5 can participate in the following haplotypes with KIR2DS1 and KIR2DS3: KIR2DL5+/KIR2DS1+ or KIR2DL5-/ KIR2DS1- and KIR2DL5+/KIR2DS3+ or KIR2DL5-/ KIR2DS3-. These results made possible the complete genotypic determination of these four KIRs. KIR2DS4 and KIR2DS5, whose number of gene copies were not fully defined in all families, presented complete LD $\left(\left|D^{\prime}\right|=1\right)$ with several other KIRs (Figure 1). Based on previously reported haplotypes [13], where KIR2DS4 is observed in complete negative LD (the presence of one gene in a given haplotype excluding the presence of the other) with KIR2DS1 and KIR2DS5 [13], we accepted that KIR2DS1 and KIR2DS4 were always inherited on different chromosomes: 2DS1+/2DS4- or 2DS1-/2DS4+. The same goes for KIR2DS4 and KIR2DS5 with only two haplotypes: 2DS4+/2DS5- or 2DS4-/2DS5+.

After these two reconstruction steps, 225 members of 67 families (patients and first degree relatives ) had known genotypes for eight KIR gene systems - 2DS2, 2DL2, 2DL3, 3DS1, 3DL1, 2DL5, 2DS3, 2DS5, 2DS1, and 2DS4 - which were used for the family-based association study.

\section{Family-based leukemia's association study}

14 different KR haplotypes were observed, which were combined in 28 KIR genotypes, 16 occurred among parents and their generation, 8 were observed only in parents' group, one was unique for leukemia patients and the remaining three genotypes were seen only in the healthy offspring. The results of the phase 2-family-based association study of KIR and KIR HLA class I ligands showed significant association between the absence of HLA-A ${ }^{\text {Bw4 }}$ alleles and leukemia $(p=0.0455)$. With respect to HLA class I haplotype, one haplotype HLA-C1 pos. C2 neg. Bw6 neg. Bw4 pos. $A^{B w 4}$ pos. $A 3 / 11$ neg. was found with $p$ value 0.038 , demonstrating its potential influence in the leukemia development.

\section{DISCUSSION}

Previous disease association studies of KIRs used solely case/control design. Family-base studies of NK receptors are carried out almost exclusively for the detailed examination of organization of the KIR region. To date, only two studies employing familybased association study design of KIRs and their ligands were conducted: one with hematology malignancies and one with Hodgkin disease [13, 19]. Ad- vantages of using a family-based association model incorporate obtaining of full KIR genetic information (number of gene copies, alleles), more accurate determination of KIR haplotypes and the inherited KIR/ HLA genotypes. Hence, in our study we included families meeting the leukemia case index. Using all available information from KIR and HLA class I ligand genotyping we were able to determine KIR profiles and the number of gene copies in 225 participants from 67 families.

Our results show an association between leukemia development and KIR/HLA class I gene systems. Although our proposed model for family-based KIR gene reconstruction was guided by the model of Besson et al. [13], it was also unique for our population, as it was constructed based on the established KIR pairwise LD adapted for Bulgarians.

In Phase 1 we primarily compared the distribution of individual KIRs, KIR genotypes, KIRA/B haplotype combinations, HLA class I ligands and KIR/ligand combinations between leukemia patients and their healthy brothers/sisters. The aim of patient/healthy sibling study was to define whether there are differences that act as genetic genetic differences that act as predetermined predisposing factors for leukemia development, assuming that leukemia affected and non-affected siblings were exposed to similar environmental factors. The comparative analysis did not show any differences in any of tested markers. However, this was not surprising, since according to Mendelian laws of inheritance probability of a pair offspring to be genetically identical is $25 \%$. Nevertheless, KIR genotype with activating/inhibitory KIR ratio of 0.57 was more frequent in the leukemia patients group than in the healthy siblings group. In this genotype, there was a higher prevalence of inhibitory KIR genes vs. activating KIR genes. Therefore, it contributes to higher leukemia susceptibility due to more inhibitory NK cell activation potential.

Phase 2: The second purpose of our research was to conduct a family-based leukemia association study to compare alleles transmittion distribution in leukemia affected offspring compared with the expected distribution of alleles among healthy offspring. For this task we developed and implemented a model for KIR gene reconstruction in families that met the leukemia case index. From the TDT analysis we found that the absence of HLA-A ${ }^{B w 4}$ ligand and the carriage of HLA-C1+/ $B^{B w 4}+/ A^{B w 4}+$ haplotype was in association with leukemia disease $(p<0.05)$. Some peculiarity should be noted in the context of HLA-C $1+/ B^{B w 4}+/ A^{B w 4}+$ haplotype. All studied individuals possessed at least one inhibitory KIR (KIR2DL2 or KIR2DL3) for 
HLA-C1 group ligand, which means that this haplotype participates in a functional inhibitory KIR/ ligand interaction. At the same time, simultaneous presence of both alleles from HLA locus binding KIR3DL1 (HLA-B ${ }^{\mathrm{Bw} 4}+n \mathrm{~A}^{\mathrm{Bw} 4}+$ ) defines more powerful inhibitory potency. On the other side the same HLA class I ligands also could interact with activating KIRs (KIR2DS2 and KIR3DS1 respectively). As a whole the frequencies of activating KIRs were lower compared to the inhibitory counterpart and we accepted that HLA-C $1+/ \mathrm{B}^{\mathrm{Bw}}+4$ $A^{B w 4}+$ haplotype should be considered as a mediator of inhibitory signals. The observed difference in HLA-A ${ }^{\mathrm{Bw} 4}$ allele distribution could not be compared with the available literature data due to the lack of such association in case/control or familybased study. Furthermore, the role of HLA-A ${ }^{\mathrm{Bw} 4}$ molecule as ligand for KIR3DL1/S1 is not fully understood and our data did not allow us to interpret the absence of HLA-A ${ }^{B w 4}$ and its role in the inhibition. Rather we can only suggest its role as a risk factor for leukemia, particularly when family history is positive for malignancy.

\section{CONCLUSION}

Although a small number of differences were reported in our study - case/control (phase 1) and family-based (phase 2), we found significant inhibitory potency variations in leukemia patients' group. The results were in accordance with our hypothesis for the role of certain KIRs and/or KIR/ ligand combinations, acting in a direction of increased NK cell inhibition or decreased activation, but more as a risk factor in leukemia susceptibility. We also considered the possible disguise of $\mathrm{KIR}$ and KIR/ligand genotypes effects from other polymorphic genes.

Conflict of interests: The authors of this paper have no conflicts of interest, including specific financial interests, relationships, and/or affiliations relevant to the subject matter or materials included.

\section{REFERENCES}

1. http://www.allelefrequencies.net/diseases/

2. Mandelboim O, Reyburn H, Vales-Gomez $M$, et al. Protection from lysis by natural killer cells of group 1 and 2 specificity is mediated by residue 80 in human histocompatibility leukocyte antigen $\mathrm{C}$ alleles and also occurs with empty major histocompatibility complex molecules. J Exp Med, 1996, $184,913-922$.
3. Collona M, Samaridis S. Cloning of immunoglobulin-superfamily members associated with HLA-C and HLA-B recognition by human natural killer cells. Science, 1995, 268: 405-408.

4. Foley B, De Santis D, Van Beelen E, et al. The reactivity of Bw4+ HLA-B and HLA-A alleles with KIR3DL1:implications for patient and donor suitability for haploidentical stem cell ransplantations. Blood, 2008, 112, 435-443.

5. Gumperz J, Litwin V, Philips J et al. The Bw4 public epitope of HLA-B molecules confers reactivity with natural killer cell clones that express NKB1, a putative HLA receptor. J Exp Med, 1995; 181, 1133-1144.

6. Hansasuta $\mathrm{P}$, Dong $\mathrm{T}$, Thananchi $\mathrm{H}$, et al. Recognition of HLAA3 and HLA-A11 by KIR3DL2 is peptide-specific. Eur J Immunol, 2004, 34, 1673-1679.

7. Biassoni $R$, Pessino A, Malaspina A, et al. Role of amino acid position 70 in the binding affinity of p50.1 and p58.1 receptors for HLA-Cw4 molecules. Eur J Immunol, 1997, 27, 3095-3099.

8. Stewart C, Laugier-Anfossi F, Vely F, et al. Recognition of peptide-MHC class I complexes by activating killer immunoglobulin-like receptors. Proc Natl Acad Sci USA, 2005, 102, 13224-13229.

9. Single R, Martin MP, Gao X, et al. Global diversity and evidence for coevolution of KIR and HLA. Nat Genet, 2007, 39, 1114-1119.

10. Alter G, Martin MP, Teigen N, et al. Differential natural killer cell-mediated inhibition of HIV-1 replication based on distinct KIR/HLA subtypes. J Exp Med, 2007, 204, 3027-3036.

11. Khakoo SI, Carrington M. KIR and disease: a model system or system of models. Immunol Rev, 2006, 214, 186-201.

12. Karabon L, Jedynak A, Giebel S, et al. KIR/HLA gene combinations influence susceptibility to B-cell chronic lymphocytic leukemia and the clinical course of disease. Tissue Antigens, 2011, 78, 129-138.

13. Besson C, Roetync S, Williams F, et al. Association of killer cell immunoglobulin-like receptor genes with Hodgkin lymphoma in a familial study. PLoS ONE, 2007, 2(5), e406.

14. Shahsavar F, Tajik N, Entezami K, et al. KIR2DS3 is associated with protection against acute myeloid leukemia. Iran J Immunol, 2010, 7, 8-17.

15. Verheyden S, Demanet C. Susceptibility to myeloid and lymphoid leukemia is mediated by distinct inhibitory KIR-HLA ligand interactions. Leukemia, 2006, 20, 1437-1438.

16. Pamuk GE, Tozkir H, Uyanik MS, et al. Natural killer cell killer immunoglobulin-like gene receptor polymorphisms in nonHodgkin lymphoma: possible association with clinical course. Leuk Lymphoma, 2015, 56(10), 2902-7.

17. Sullivan EM, Jeha S, Kang G, et al. NK cell genotype and phenotype at diagnosis of acute lymphoblastic leukemia correlate with postinduction residual disease. Clin Cancer Res, 2014, 20(23), 5986-94.

18. Misra MK, Prakash S, Moulik NR, et al. Genetic associations of killer immunoglobulin like receptors and class I human leukocyte antigens on childhood acute lymphoblastic leukemia among north Indians. Hum Immunol, 2016, 77(1), 41-6.

19. Sugioka DK, Gonçalves CE, Bicalho MD. KIR repertory in patients with hematopoietic diseases and healthy family members. BMC Hematology, 2016, 16, 25. 
20. https://www.ebi.ac.uk/ipd/kir/

21. Varbanova V, Mihaylova A, Naumova E. Olerup SSP KIR HLA ligand typing Kit. A new opportunity for KIR ligand assignment in clinical settings. C R Acad Bulg Sci, 2010, 63(5), 713-722.

22. Gonzalez-Galarza F, Christmas S, Middleton D, Jones A. Allele frequency net: a database and online repository for immune gene frequencies in worldwide populations. Nucleic Acids Research, 2011, 39, D913-D919.

23. Spielman RS, McGinnis RE, Ewens WJ. Transmission test for linkage disequilibrium: the insulin gene region and insulindependent diabetes mellitus (IDDM). Am J Hum Genet, 1993, 52: 506-516.

24. Wall J, Pritchard J. Haplotypes blocks and linkage disequilibrium in the human genome. Nat Rev Genet, 2003, 4, 587-597.

25. Knapp M. The transmission/disequilibrium test and parental-genotype reconstruction: the reconstruction-combined transmission/disequilibrium test. Am J Hum Genet, 1999, 64, 861-870.
26. Spielman RS, Ewens WJ. A sibship test for linkage in the presence of association: the sib transmission/disequilibrium test. Am J Hum Genet, 1998, 62, 450-458.

27. Horvath $S, X u X$, Laird NM. The family based association test method: strategies for studying general genotype-phenotype associations. Eur J Hum Genet, 2001, 9, 301-306.

28. Faure M, Longo E. KIR2DL4 (CD158d), an NK cell-activating receptor with inhibitory potential. J Immunol, 2002, 168, 6208-14.

29. Kikuchi-Maki A, Yusa S, Catina T, Cambpbell K. KIR2DL4 is an IL-2 regulated NK cells receptor that exhibits limited expression in humans but triggers strong IFN-gamma production. J Immunol, 2003, 171, 3415-3425.

30. Hsu K, Chida S, Geraghty D and Dupont B. The killer cell immunoglobulin-like receptor (KIR) genomic region: geneorder, haplotypes and allelic polymorphism. Immunological Reviews, 2002, 190, 40-52.

Received: January, 2019 - Revised: February, 2019 Accepted: April, 2019 